Reprod. Nutr. Dévelop., 1988, 28 (1), 199-200.

\title{
Influence de deux environnements climatiques contrastés sur le besoin énergétique d'entretien de la brebis tarie
}

\author{
F. BOCQUIER, A. BRELURUT, M. THERIEZ \\ avec la collaboration technique de $H$. CASSAGNES et P. LEBECQUE
}

Laboratoire de la Production Ovine,

I.N.R.A., Theix, 63122 Ceyrat, France.

Summary. In our experimental conditions there was a large difference $(35-51 \%)$ in maintenance energy requirements of ewes (MEm) between individually fed ewes in a closed barn at $19^{\circ} \mathrm{C}$ : normal experimental conditions $\left(80 \mathrm{kcal} \mathrm{ME} / \mathrm{kg} \mathrm{BW} \mathrm{B}^{0,75} / \mathrm{d}\right)$ and group fed ewes in an open barn at $3^{\circ} \mathrm{C}$ : normal winter rearing conditions $1114 \mathrm{kcal} \mathrm{ME} / \mathrm{kg}$ $\left.\mathrm{BW}^{0,75} / \mathrm{d}\right)$.

Des bilans énergétiques réalisés à la suite d'essais en cases individuelles, en bergerie fermée, aussi bien avec des brebis allaitantes (Tissier et al., 1979) qu'avec des brebis traites (Vermorel et al., 1987) conduisent à des estimations des besoins énergétiques d'entretien plus faibles que ceux retenus par I'I.N.R.A. en 1978 : $95 \mathrm{kcal} \mathrm{d}^{\prime e ́ n e r g i e ~ m e ́ t a b o l i s a b l e ~} / \mathrm{kg} \mathrm{PV}^{0,75} /$ jour : EMm*. II était donc important d'évaluer la différence de besoins d'entretien des brebis entre nos conditions expérimentales et une conduite d'élevage plus classique.

Matériel et méthodes. De février à avril, 11 brebis de race Limousine, récemment taries et non tondues (toison de 10 à $12 \mathrm{~cm}$ ) ont été réparties entre deux environnements contrastés. Cinq brebis ont été placées en bergerie (INT) chauffée $\left(\mathrm{Tm}=19^{\circ} \mathrm{C}\right)$, en cases individuelles. Les autres ont été placées en bergerie ouverte (EXT) en un lot de 6 brebis $\left(\mathrm{Tm}=2,9 \pm 4,8^{\circ} \mathrm{C}\right)$ dont les consommations n'ont pas pu être individualisées.

Toutes les brebis ont reçu la même quantité d'une ration constituée de fourrages secs et d'aliment concentré comportant $12 \%$ de matières azotées et $18 \%$ de cellulose brute ( $\%$ MS). La digestibilité de l'énergie brute (EB) de la ration, mesurée sur 4 brebis à $19^{\circ} \mathrm{C}$, a été de 0,783 , avec $\mathrm{EM} / \mathrm{EB}=0,648$. La ration a apporté $180 \mathrm{kcal} \mathrm{EM}^{*}$ et $109 \mathrm{~g}$ de $\mathrm{MAD} / \mathrm{jour}(149 \mathrm{~g} \mathrm{PDI})$, soit respectivement 1,9 et 1,8 fois les besoins d'entretien théoriques des brebis (I.N.R.A., 1978). Les brebis, en phase de reconstitution de leurs réserves corporelles, ont été maintenues en expérience jusqu'à ce qu'elles gagnent 8 à $10 \mathrm{~kg}$ de poids vif dans les deux environnements. Les variations d'énergie corporelle ont été mesurées par la méthode de l'espace de diffusion de l'eau lourde (Bocquier et Thériez, 1984).

Résultats et discussion. Pour des ingestions quotidiennes d'EM (EMI) très voisines, le poids vif moyen a augmenté de $11 \mathrm{~kg}$ en 42 jours pour les brebis INT et de $7,9 \mathrm{~kg}$ en 75 jours pour le lot EXT (tabl. 1). Les brebis du lot INT ont déposé quotidiennement $61 \mathrm{kcal}^{*} \mathrm{~d}^{\prime}$ énergie corporelle $(E C)$ contre 38 pour celles du lot EXT $(P<0,05)$. 
On peut penser que le rendement $k$ de l'utilisation de l'EM pour le dépôt d'énergie corporelle : $E C=k \times(E M l-E M m)$ est proche de $k l$ au début de la période puisque les brebis viennent d'être taries (Vermorel et al., 1987). II diminuerait ensuite pour se rapprocher de kf. Pour les brebis du lot INT qui ne sont restées que 42 jours en expérimentation nous avons retenu $\mathrm{k}=\mathrm{kl}$; EMm est alors égal à $80^{*}$, soit $84 \%$ du besoin théorique (I.N.R.A., 1978), ce qui est en accord avec les résultats des essais antérieurs. En revanche, pour les brebis du lot EXT, EMm devrait se situer entre $120^{*}$ (avec $k=k \mathrm{l}$ ) et $108^{*}(\mathrm{k}=\mathrm{kf}$ ) soit 126 et $114 \%$ du besoin théorique (cf. tabl. 1).

TABL. 1. - Poids vifs et bilans énergétiques de brebis conduites dans deux environnements contrastés, bergerie fermée (INT) ou ouverte (EXT).

\begin{tabular}{|c|c|c|c|}
\hline Lot expérimental & INT & EXT & Ecart-type résiduel \\
\hline Nombre de brebis & 5 & 6 & \\
\hline Poids vif initial $(\mathrm{kg})$ & 63,8 & 67,9 & 3,8 \\
\hline \multirow{2}{*}{$\begin{array}{l}\text { Variation de poids vif }(\mathrm{kg}) \\
\text { en } 42 j \\
\text { en } 75 i\end{array}$} & & & \\
\hline & $\begin{array}{c}+11,0 \\
-\end{array}$ & $\begin{array}{l}+4,0 \\
+7,9\end{array}$ & $\begin{array}{l}- \\
-\end{array}$ \\
\hline Durée de l'essai (jours) & 42 & 75 & \\
\hline EM ingérée (kcal/kg $\mathrm{P}^{0,75 / \mathrm{j})}$ & 179 & 182 & 6,4 \\
\hline$E$ déposée $\left(\mathrm{kcal} / \mathrm{kg} \mathrm{P}^{0,75} / \mathrm{j}\right)$ & 61 & 38 & 12,9 \\
\hline \multicolumn{4}{|l|}{$\begin{array}{l}\text { Besoin d'entretien (EMm) } \\
\text { (kcal/kg } \mathrm{P} 0,75 / \mathrm{j})\end{array}$} \\
\hline avec $\mathrm{kl}=0,618$ (I.N.R.A., 1978) & 80 & 120 & - \\
\hline avec kf $=0,511$ (I.N.R.A., 1978) & 59 & 108 & - \\
\hline
\end{tabular}

Même si nous n'avons pas pu faire la part de l'accroissement des dépenses énergétiques dues à l'activité physique des brebis, nous observons une différence de 35 à $51 \%$ de l'EMm entre les deux environnements $\left(19\right.$ et $\left.3^{\circ} \mathrm{C}\right)$. Ce résultat est conforme à l'accroissement de la production de chaleur $(+58 \%)$ de moutons moyennement lainés, lorsque la température ambiante passe de +14 à - $10^{\circ} \mathrm{C}$ (Webster et Blaxter, 1966). En pratique, l'accroissement de l'EMm des brebis placées dans des conditions difficiles pourrait être en partie compensée par l'accroissement de la consommation de fourrage (20 à $30 \%$ ) comme nous l'avons observé sur des béliers à l'entretien.

Bocquier F., Thériez M., 1984. Proc. E.E.C., Bristol Dec. 1983, Eds D. Lister, Elsevier, 152-157. I.N.R.A., 1978. In Alimentation des Ruminants. I.N.R.A. Publ. Ed., Versailles, 229-244.

Tissier M., Thériez M., Purroy A., Brelurut A., 1979. Proc. VII/th int. Symp. Energy Metabolism, Cambridge, Sept. 1979, 329-333.

Vermorel M., Bocquier F., Vernet J., Brelurut A., 1987. Proc. Xth int. Symp. Energy Metabolism, Arlie, U.S.A., 314-317.

Webster A. J. F., Blaxter K. L., 1966. Res, vet. Sci., 7, 454-465. 